\title{
SOURCE-DESTINATION FLOW ON A ROAD NETWORK*
}

\author{
MAURO GARAVELLO ${ }^{\dagger}$ AND BENEDETTO PICCOLI ${ }^{\ddagger}$
}

\begin{abstract}
We construct a model of traffic flow with sources and destinations on a roads network. The model is based on a conservation law for the density of traffic and on semilinear equations for traffic-type functions, i.e. functions describing paths for cars.

We propose a definition of solution at junctions, which depends on the traffic-type functions. Finally we prove, for every positive time $T$, existence of entropic solutions on the whole network for perturbations of constant initial data.

Our method is based on the wave-front tracking approach.
\end{abstract}

Key words. Scalar conservation law, semilinear equation, traffic flow.

AMS subject classifications. 90B20, 35L65.

\section{Introduction}

This paper deals with a model of traffic flow on a road network with sources and destinations, that are, respectively, areas from which cars start their travels and areas where they end. A road network is a finite collection of roads, modeled by closed intervals of $\mathbb{R}$, connected together by junctions.

On each road, we consider the car density $\rho$ and a vector $\pi$ describing the traffic types, i.e. the percentages of cars going from a fixed source to a fixed destination. For the evolution of the density $\rho$, we use the fluidodynamic model proposed independently by Lighthill and Whitham [16] in 1955 and by Richards [17] in 1956. It is based on the conservation of cars and so it is described by the equation

$$
\rho_{t}+f(\rho)_{x}=0
$$

where the density $\rho(t, x)$ belongs to $\left[0, \rho_{\max }\right]$ and the flux $f(\rho)$ is given by $\rho v$, where $v$ is the average speed of cars. More complex models were also proposed in $[5,9,12,13]$.

To deal with the big number of roads, it is customary in transportation sciences to consider complex networks. However, the first papers treating fluidodynamic models on a network (and not just on one road) were $[7,8,11,14,15]$. In particular, [11] is based on a second order model proposed by Aw and Rascle [3] in 2000. All these papers determine the behavior at junctions depending on the car density on each road and some given parameters. A more accurate model must take into account that drivers choose a given route depending on their initial and final address. The idea of sources and destinations was already proposed in 1965; see [10]. In this paper, we develop this idea for the fluidodynamic model proposed by Lighthill, Whitham and Richards. This means that cars have a precise path inside the network. Such paths are determined by the behavior at junctions via the coefficients $\pi$.

It is easy to understand that, in each road, the evolution of $\pi$ follows a semilinear equation

$$
\pi_{t}+v(\rho) \pi_{x}=0
$$

${ }^{*}$ Received: February 27, 2005; accepted (in revised version): May 18, 2005. Communicated by Lorenzo Pareschi.

${ }^{\dagger}$ Dipartimento di Matematica e Applicazioni, Università degli Studi di Milano-Bicocca, Via R. Cozzi 53 - Edificio U5 - 20125 Milano, Italy (mauro.garavello@unimib.it). Partially supported by Istituto per le Applicazioni del Calcolo "Mauro Picone" and by Università di Roma "La Sapienza".

$\ddagger$ I.A.C., Viale del Policlinico 137, 00161 Roma, Italy (piccoli@iac.cnr.it). 
hence inside roads the evolution of $\pi$ is influenced by the average speed of cars, which we suppose to be a strictly decreasing function of the density.

In [8], Riemann problems at junctions were solved by means of two rules:

(A) cars distribute on outgoing roads according to fixed percentages;

(B) respecting rule (A), the flux is maximized at junctions.

It was proved that $(\mathrm{A})$ and $(\mathrm{B})$ isolate a unique solution and Cauchy problems were solved via wave-front tracking. Rule (A) can be described by a matrix $A=\left(\alpha_{j i}\right)$, where the index $i$ runs over the incoming roads of the junction and the index $j$ runs over the outgoing roads. In the present paper, the matrix $A$ is determined via the coefficients $\pi$ and the rule (B) is replaced by another maximization procedure. The latter takes into account priorities over incoming roads and ensures continuity of solutions with respect to the coefficients $\pi$ (not granted for the solution chosen in [8]).

Then, to construct a solution on the whole network, we use also in this case a wave-front tracking method; see [6]. The key point is to derive some BV estimates on the piecewise constant approximate solutions, in order to have convergence. Unfortunately it happens that large $\pi$-variations may occur at junctions (produced by $\rho$-waves of small amplitude). Thus we are able to prove existence of solutions only for small BV initial data (as it occurs for systems of conservation laws).

The paper is organized as follows. In Section 2 we give the basic definition for our model and we describe admissible conditions for the network. In Section 3 we describe in detail the solution of the Riemann problem at junctions and we also do a comparison with the Riemann problem introduced in [7] and in [8]. In Section 4, we briefly describe the wave-front tracking method, in Section 5 we give estimates on the interactions between waves. Finally Section 6 deals with the existence of solutions.

\section{Basic definitions}

We introduce some basic notations and recall some results from $[7,8]$.

Definition 2.1. A network is given by a \%-tuple $(\mathcal{I}, \mathcal{F}, \mathcal{J}, \mathcal{S}, \mathcal{D}, \mathcal{R}, \mathcal{P})$ where:

Edges $\mathcal{I}$ is a finite collection of intervals, called roads, $I_{i}=\left[a_{i}, b_{i}\right] \subseteq \mathbb{R}, i=1, \ldots, N$; Fluxes $\mathcal{F}$ is a finite collection of fluxes $f^{i}:\left[0, \rho_{\text {max }}^{i}\right] \mapsto \mathbb{R}$;

Junctions $\mathcal{J}$ is a finite collection of subsets of $\{ \pm 1, \ldots, \pm N\}$. If $j \in J \in \mathcal{J}$, then the road $I_{|j|}$ is crossing at $J$ as entering road (i.e. at point $b_{i}$ ) if $j>0$ and as exiting road (i.e. at point $a_{i}$ ) if $j<0$. For each junction $J \in \mathcal{J}$, we indicate by $\operatorname{Inc}(J)$ the set of incoming roads, that are $I_{i}$ 's such that $i \in J$ and $i>0$, while by $\operatorname{Out}(J)$ the set of outgoing roads, that are $I_{i}$ 's such that $-i \in J$ and $i>0$;

Sources $\mathcal{S}$ is a finite subset of $\{1, \ldots, N\}$, representing roads connected to traffic sources;

Destinations $\mathcal{D}$ is a finite subset of $\{1, \ldots, N\}$, representing roads leading to final destinations;

Traffic distribution functions $\mathcal{R}$ is a finite collection of functions $r_{J}: \operatorname{Inc}(J) \times \mathcal{S} \times$ $\mathcal{D} \rightarrow \operatorname{Out}(J)$;

Right of way parameters $\mathcal{P}$ is a finite collection of vectors $P_{J} \in \mathbb{R}^{l}$ and $l=$ $\sharp \operatorname{Inc}(J)-1$.

The meanings of roads, fluxes, junctions, sources and destinations are clear. Each flow distribution function $r_{J}$ indicates the direction at the junction $J$ of traffic that started at source $s$ and has $d$ as final destination. Notice that we need $\operatorname{Inc}(J) \neq \emptyset$ and $\operatorname{Out}(J) \neq \emptyset$ in order $r_{J}$ to be well defined, and we give additional conditions 
later to have a suitable network. The right of way parameters determine a level of "importance" at the junctions of incoming roads.

On each road $I_{i}$, we consider the evolution equation

$$
\rho_{t}^{i}+f^{i}\left(\rho^{i}\right)_{x}=0 .
$$

Hence the datum on the network is given by a finite collection of functions $\rho^{i}$ : $\left[0,+\infty\left[\times I_{i} \mapsto\left[0, \rho_{\max }^{i}\right], i=1, \ldots, N\right.\right.$.

Solutions to equation (2.1) may develop discontinuities, thus one considers weak solutions in the sense of distributions. On each road $I_{i}$ we want $\rho^{i}$ to be a weak entropic solution, that is, for every smooth function $\varphi:] 0,+\infty\left[\times I_{i} \rightarrow \mathbb{R}\right.$ with compact support on $] 0,+\infty[\times] a_{i}, b_{i}[$,

$$
\int_{0}^{+\infty} \int_{a_{i}}^{b_{i}}\left(\rho^{i} \frac{\partial \varphi}{\partial t}+f^{i}\left(\rho^{i}\right) \frac{\partial \varphi}{\partial x}\right) d x d t=0
$$

and for every $k \in \mathbb{R}$ and $\tilde{\varphi}:] 0,+\infty\left[\times I_{i} \rightarrow \mathbb{R}\right.$ smooth, positive with compact support on ] $0,+\infty[\times] a_{i}, b_{i}[$

$$
\int_{0}^{+\infty} \int_{a_{i}}^{b_{i}}\left(\left|\rho^{i}-k\right| \frac{\partial \tilde{\varphi}}{\partial t}+\operatorname{sgn}\left(\rho^{i}-k\right)\left(f^{i}\left(\rho^{i}\right)-f^{i}(k)\right) \frac{\partial \tilde{\varphi}}{\partial x}\right) d x d t \geq 0 .
$$

Classical theory of conservation laws, see [6], ensures, for every initial data in $L^{1}$, the existence of a weak entropic solution to $(2.1)$ on $\mathbb{R}$. For initial data in $L^{1} \cap L^{\infty}$ solutions depend in a Lipschitz continuous way in the $L^{1}$ norm.

For each $i \in \mathcal{S}$ (resp. $i \in \mathcal{D}$ ) and measurable $\psi^{i}:\left[0,+\infty\left[\rightarrow\left[0, \rho_{\text {max }}^{i}\right]\right.\right.$ we consider solutions verifying the boundary condition $\rho^{i}\left(t, a_{i}\right)=\psi^{i}(t)\left(\operatorname{resp} . \rho^{i}\left(t, b_{i}\right)=\psi^{i}(t)\right)$ in the sense of [4].

2.1. Traffic distribution at junctions. The evolution of car densities $\rho^{i}$ is thus described on roads and at sources and destinations. To treat the evolution at junctions, we introduce some definitions.

Fix a junction $J$ and assume for notational simplicity that $\operatorname{Inc}(J)=\{1, \ldots, n\}$ and $\operatorname{Out}(J)=\{n+1, \ldots, n+m\}$.

DEFINITION 2.2. A weak solution at the junction $J$ is a collection of functions $\rho^{i}$ : $\left[0,+\infty\left[\times I_{i} \rightarrow\left[0, \rho_{\text {max }}^{i}\right], i=1, \ldots, n+m\right.\right.$, satisfying

$$
\sum_{l=0}^{n+m}\left(\int_{0}^{+\infty} \int_{a_{l}}^{b_{l}}\left(\rho^{l} \frac{\partial \varphi_{l}}{\partial t}+f^{l}\left(\rho^{l}\right) \frac{\partial \varphi_{l}}{\partial x}\right) d x d t\right)=0
$$

for each $\varphi_{1}, \ldots, \varphi_{n+m}$ smooth having compact support in $] 0,+\infty[\times \mathbb{R}$, that are also smooth across the junction, i.e.

$$
\varphi_{i}\left(\cdot, b_{i}\right)=\varphi_{j}\left(\cdot, a_{j}\right), \quad \frac{\partial \varphi_{i}}{\partial x}\left(\cdot, b_{i}\right)=\frac{\partial \varphi_{j}}{\partial x}\left(\cdot, a_{j}\right),
$$

for every $i=1, \ldots, n$, and $j=n+1, \ldots, n+m$.

REMARK 2.1. Let $\rho$ be a weak solution at a junction $J$ and assume that each $x \mapsto \rho^{i}(t, x)$ has bounded variation. We can deduce that it satisfies the RankineHugoniot Condition at the junction $J$, namely

$$
\sum_{i=1}^{n} f^{i}\left(\rho^{i}\left(t, b_{i}-\right)\right)=\sum_{j=n+1}^{n+m} f^{j}\left(\rho^{j}\left(t, a_{j}+\right)\right),
$$


for almost every $t>0$.

Definition 2.3. A traffic-type function on a road $I_{i}$ is a function

$$
\pi^{i}:\left[0, \infty\left[\times\left[a_{i}, b_{i}\right] \times \mathcal{S} \times \mathcal{D} \rightarrow[0,1]\right.\right.
$$

such that, for every $t \in\left[0, \infty\left[\right.\right.$ and $x \in\left[a_{i}, b_{i}\right]$,

$$
\sum_{s \in \mathcal{S}, d \in \mathcal{D}} \pi^{i}(t, x, s, d)=1
$$

Hence $\pi^{i}(t, x, s, d)$ specifies the amount of the density $\rho^{i}(t, x)$ that started from source $s$ and is moving towards the final destination $d$.

Let us now show how a solution at the junction $J$ is constructed using $\pi$ and $r_{J}$.

Fix a time $t$ and assume that for all $i \in \operatorname{Inc}(J), s \in \mathcal{S}$ and $d \in \mathcal{D}, \pi^{i}(t, \cdot, s, d)$ admits a limit at the junction $J$, i.e. left limit at $b_{i}$. For $i \in\{1, \ldots, n\}, j \in\{n+1, \ldots, n+m\}$, set

$$
\alpha_{j, i}=\sum_{s \in \mathcal{S}, d \in \mathcal{D}, r_{J}(i, s, d)=j} \pi^{i}\left(t, b_{i}-, s, d\right) .
$$

Notice that $\alpha_{j, i}$ is the amount of the density $\rho^{i}$ that flows toward road $I_{j}$. From the definition of traffic-type functions we get:

$$
\alpha_{j, i} \in[0,1], \quad \sum_{j \in \operatorname{Out}(J)} \alpha_{j, i}=1 .
$$

The fluxes $f^{j}\left(\rho^{j}\right)$ to be consistent with the traffic-type functions must satisfy the following relations:

$$
f^{j}\left(\rho^{j}\left(\cdot, a_{j}+\right)\right)=\sum_{i=1}^{n} \alpha_{j, i} f^{i}\left(\rho^{i}\left(\cdot, b_{i}-\right)\right),
$$

for each $j=n+1, \ldots, n+m$. However this is not sufficient to determine a unique solution. Hence we introduce the concept of admissible weak solution, using also the right of way parameters.

Definition 2.4. Let $\rho=\left(\rho^{1}, \ldots, \rho^{n+m}\right)$ be such that $\rho^{i}(t, \cdot)$ is of bounded variation for every $t \geq 0$. Then $\rho$ is an admissible weak solution of (1.1) at the junction $J$ related to the matrix $A$ and to the right of way parameters $P_{J}=\left(p_{1}, \ldots, p_{n-1}\right) \in \mathbb{R}_{+}^{n-1}$ if and only if the following properties hold:

(i) $\rho$ is a weak solution at the junction $J$;

(ii) $f^{j}\left(\rho^{j}\left(\cdot, a_{j}+\right)\right)=\sum_{i=1}^{n} \alpha_{j, i} f^{i}\left(\rho^{i}\left(\cdot, b_{i}-\right)\right)$, for each $j=n+1, \ldots, n+m$;

(iii)

$$
c_{2} \sum_{i=1}^{n} f^{i}\left(\rho^{i}\left(\cdot, b_{i}-\right)\right)-c_{1}\left[\operatorname{dist}\left(\left(f^{1}\left(\rho^{1}\left(\cdot, b_{1}-\right)\right), \ldots, f^{n}\left(\rho^{n}\left(\cdot, b_{n}-\right)\right)\right), r\right)\right]^{2}
$$

is maximum subject to (ii), where $c_{1}$ and $c_{2}$ are strictly positive constants, and $\operatorname{dist}(\cdot, r)$ denotes the euclidean distance in $\mathbb{R}^{n}$ from the line $r$, which is given by

$$
\left\{\begin{array}{l}
\gamma_{n}=p_{1} \gamma_{1}, \\
\vdots \\
\gamma_{n}=p_{n-1} \gamma_{n-1} .
\end{array}\right.
$$


REMARK 2.2. Condition (2.5) is automatically guaranteed by condition (2.7).

Conditions (i) and (ii) do not suffice to solve Riemann problems at junctions in a unique way. Thus one has to impose some modelling assumptions. A further possibility could be to impose

$$
\left(f^{1}\left(\rho^{1}\left(\cdot, b_{1}-\right)\right), \ldots, f^{n}\left(\rho^{n}\left(\cdot, b_{n}-\right)\right)\right) \in r
$$

and then to maximize with respect to a free parameter. Unfortunately this choice is not realistic. In fact, let us consider a simple junction with 2 incoming roads and 1 outgoing road. If the outgoing road and one incoming road are empty, then just the origin in the $\left(\gamma_{1}, \gamma_{2}\right)$ plane satisfies the previous conditions. Therefore for this model, no car passes through the junction, which is clearly anti intuitive.

If we substitute property (iii) of Definition 2.4 with the following

(iii') $\sum_{i=1}^{n} f^{i}\left(\rho^{i}\left(\cdot, b_{i}-\right)\right)$ is maximum subject to (ii),

i.e. we are considering the same solution as in [8], then uniqueness is not granted. In fact condition $(\mathrm{C})$ in [8], necessary for uniqueness, may fail. Moreover, with this choice, the solution for the fluxes does not depend in a continuous way by the matrix $A$, which is the case for our quadratic maximization.

2.2. Evolution equations for traffic-type functions. We assume the following. inside each road $I_{i}$, cars move at some averaged speed $v^{i}$ that depends on the local density $\rho^{i}$. Moreover $v^{i}$ is independent from $\pi$. In this case the flux function is given by:

$$
f^{i}\left(\rho^{i}\right)=v^{i}\left(\rho^{i}\right) \rho^{i}
$$

If $x(t)$ denotes a trajectory of a car inside the road $I_{i}$, then we get

$$
\pi^{i}(t, x(t), s, d)=\text { const. }
$$

Taking the total differential with respect to the time, we deduce the semilinear equation:

$$
\partial_{t} \pi^{i}(t, x, s, d)+\partial_{x} \pi^{i}(t, x, s, d) \cdot v^{i}\left(\rho^{i}(t, x)\right)=0 .
$$

This equation is coupled with equation (2.1). More precisely on road $I_{i}$ equation (2.8) depends on the solution of (2.1), while in turn at junctions the values of $\pi^{i}$ determine the traffic distribution on outgoing roads as explained in the previous section.

2.3. Admissible networks and solutions. Let us now give some admissibility conditions on the network.

Definition 2.5. A network $(\mathcal{I}, \mathcal{F}, \mathcal{J}, \mathcal{S}, \mathcal{D}, \mathcal{R}, \mathcal{P})$ is admissible if the following conditions hold.

1. Each $f^{i} \in \mathcal{F}$ is given by $f^{i}\left(\rho^{i}\right)=v^{i}\left(\rho^{i}\right) \rho^{i}$, where $v^{i}$, the velocity field, is smooth, strictly decreasing, and $v^{i}\left(\rho_{\max }^{i}\right)=0$.

2. Each $f^{i} \in \mathcal{F}$ is a smooth strictly concave function with

$$
f^{i}(0)=f^{i}\left(\rho_{\text {max }}^{i}\right)=0 .
$$

Thus there exists a unique $\left.\sigma^{i} \in\right] 0, \rho_{\text {max }}^{i}\left[\right.$ global maximum with $\left(f^{i}\right)^{\prime}\left(\sigma^{i}\right)=0$. 
3. Each $i \in\{ \pm 1, \ldots, \pm N\}$ belongs to at most one $J \in \mathcal{J}$.

4. For each $i \in\{1, \ldots, N\}$, exactly one of the following cases happens:

(a) there exists $J \in \mathcal{J}$ such that $i \in J \cap \mathcal{S}$;

(b) there exists $J \in \mathcal{J}$ such that $-i \in J$ and $i \in \mathcal{D}$;

(c) there exist $J, J^{\prime} \in \mathcal{J}$ such that $i \in J$ and $-i \in J^{\prime}$.

5. $\mathcal{S} \cap \mathcal{D}=\emptyset$.

6. For every $\left.J \in \mathcal{J}, P_{J} \in\right] 0,+\infty[l$, where $l=\sharp \operatorname{Inc}(J)-1$.

7. For every $s \in \mathcal{S}$ and $d \in \mathcal{D}$, the functions $r_{J}$ determine a unique path, that is a finite sequence of roads-junctions $I_{i_{1}}, J_{l_{1}}, \cdots, I_{i_{k}}, J_{l_{k}}, I_{i_{k+1}}$ such that

(a) $i_{1}=s, i_{k+1}=d$;

(b) $i_{h} \in \operatorname{Inc}\left(J_{l_{h}}\right)$ for every $h \in\{1, \ldots, k\}$;

(c) $J_{l_{h}} \neq J_{l_{h^{\prime}}}$ for every $h, h^{\prime} \in\{1, \ldots, k\}, h \neq h^{\prime}$;

(d) $r_{J_{h}}\left(i_{h}, s, d\right)=i_{h+1}$ for every $h \in\{1, \ldots, k\}$.

REMARK 2.3. The first two conditions in the previous definition are needed to have consistency to the model. In particular we assume that the speed of cars is decreasing with respect to the quantity of cars in roads and is equal to zero when the density is maximum; hence the flux must be zero if the density is maximum. For some traffic model $v^{i}$ may explode at 0 . Moreover the concavity condition of the flux implies that the speed of $\rho$-waves may vary in the interval $\left[f^{i^{\prime}}\left(\rho_{\max }^{i}\right), f^{i^{\prime}}(0)\right]$.

Conditions 3. and 4. imply that each road is connected at least with one junction and that each road either can be connected with a source and a junction, or can be connected with a destination and a junction or finally can be connected with two junctions.

Condition 5. ensures to avoid path triviality.

Condition 6 . gives admissible weights for priorities of incoming roads of junctions.

Finally condition 7 . implies existence and uniqueness for paths connecting each source and each destination.

Given an admissible network we have to specify how to define a solution in relation to sources, destinations and junctions. For sources and junctions it is enough to use the same definition of [4].

Definition 2.6. Consider an admissible network $(\mathcal{I}, \mathcal{F}, \mathcal{J}, \mathcal{S}, \mathcal{D}, \mathcal{R}, \mathcal{P})$. A set of Initial-Boundary Conditions (briefly $I B C$ ) is given assigning measurable functions $\bar{\rho}^{i}: I_{i} \rightarrow\left[0, \rho_{\text {max }}^{i}\right], \bar{\pi}^{i}:\left[a_{i}, b_{i}\right] \times \mathcal{S} \times \mathcal{D} \rightarrow[0,1], i=1, \ldots, N$ and measurable functions $\psi^{i}:\left[0,+\infty\left[\rightarrow\left[0, \rho_{\text {max }}^{i}\right], i \in \mathcal{S} \cup \mathcal{D}\right.\right.$ with the property that for every $s \in \mathcal{S}$ and $d \in \mathcal{D}$, $\bar{\pi}^{i}(\cdot, s, d)=0$ whenever $I_{i}$ does not belong to the path for the source $s$ and destination $d$ defined at the point 7 of Definition 2.5.

Definition 2.7. Consider an admissible network $(\mathcal{I}, \mathcal{F}, \mathcal{J}, \mathcal{S}, \mathcal{D}, \mathcal{R}, \mathcal{P})$ and a set of IBC. A set of functions $\rho=\left(\rho^{1}, \ldots, \rho^{N}\right)$ with $\rho^{i}:\left[0,+\infty\left[\times I_{i} \rightarrow\left[0, \rho_{\text {max }}^{i}\right]\right.\right.$ continuous as functions from $\left[0,+\infty\left[\right.\right.$ into $L^{1}$, and $\Pi=\left(\pi^{1}, \ldots, \pi^{N}\right)$ with $\pi^{i}:\left[0,+\infty\left[\times I_{i} \times \mathcal{S} \times \mathcal{D} \rightarrow\right.\right.$ $[0,1]$, continuous as functions from $\left[0,+\infty\left[\right.\right.$ into $L^{1}$ for every $s \in \mathcal{S}, d \in \mathcal{D}$, is an admissible solution if the following holds. Each $\rho^{i}$ is a weak entropic solution to (2.1) on $I_{i}$, $\rho^{i}(0, x)=\bar{\rho}^{i}(x)$ for almost every $x \in\left[a_{i}, b_{i}\right], \rho^{i}\left(t, a_{i}\right)=\psi^{i}(t)$ if $i \in \mathcal{S}$ and $\rho^{i}\left(t, b_{i}\right)=\psi^{i}(t)$ if $i \in \mathcal{D}$ in the sense of [4]. Each $\pi^{i}$ is a weak solution to the corresponding equation (2.8). Finally at each junction $\rho$ is a weak solution and is an admissible weak solution for $\Pi$ in case of bounded variation.

Regarding sources and destinations, the treatment of boundary data in the sense of [4] can be done in the same way as in $[1,2]$. Thus we treat the construction of solutions only inside the network. 


\section{The Riemann Problem}

In this section we consider solutions to Riemann problems at junctions. This is the basic ingredient to define a wave-front tracking algorithm to construct solutions.

A Riemann problem is a Cauchy problem for an initial datum of Heaviside type, that is piecewise constant with only one discontinuity. Such solutions are formed by continuous waves called rarefactions and by traveling discontinuities called shocks. The speed of waves are related to the values of $f^{\prime}(\rho)$. Entropic solutions to Riemann problems on roads are described for example in [6].

We need to define a solution for a Riemann problem at junctions. A Riemann problem at a junction is a Cauchy problem with constant initial data on each road of the junction. Moreover a Riemann solver at a junction is a function which gives, for each initial condition, a solution to the corresponding Riemann problem.

Consider a junction $J$ in which there are $n$ roads with incoming traffic and $m$ roads with outgoing traffic. For simplicity we indicate by

$$
(t, x) \in \mathbb{R}_{+} \times I_{i} \mapsto \rho^{i}(t, x) \in\left[0, \rho_{\text {max }}^{i}\right], \quad i=1, \ldots, n,
$$

the densities of the cars on the road with incoming traffic and

$$
(t, x) \in \mathbb{R}_{+} \times I_{j} \mapsto \rho^{j}(t, x) \in\left[0, \rho_{\text {max }}^{j}\right], \quad j=n+1, \ldots, n+m,
$$

those on the roads with outgoing traffic.

Without loss of generality, we assume that the fluxes $f^{i}, f^{j}(i \in\{1, \ldots, n\}, j \in\{n+$ $1, \ldots, n+m\}$ ) are all the same and we indicate them with $f$. Hence we assume $\rho_{\max }^{i}=$ $\rho_{\text {max }}^{j}=1$ and we have $\sigma=\sigma^{i}=\sigma^{j}$ for every $i \in\{1, \ldots, n\}$ and $j \in\{n+1, \ldots, n+m\}$.

We need some more notation:

Definition 3.1. Let $\tau:[0,1] \rightarrow[0,1]$ be the map such that:

1. $f(\tau(\rho))=f(\rho)$ for every $\rho \in[0,1]$;

2. $\tau(\rho) \neq \rho$ for every $\rho \in[0,1] \backslash\{\sigma\}$.

Clearly, $\tau$ is well defined and satisfies

$$
0 \leq \rho \leq \sigma \Longleftrightarrow \sigma \leq \tau(\rho) \leq 1, \quad \sigma \leq \rho \leq 1 \Longleftrightarrow 0 \leq \tau(\rho) \leq \sigma
$$

ThEOREM 3.2. Let $(\mathcal{I}, \mathcal{F}, \mathcal{J}, \mathcal{S}, \mathcal{D}, \mathcal{R}, \mathcal{P})$ be an admissible network and $J$ a junction with $n$ incoming roads and $m$ outgoing ones. For every $\rho_{1,0}, \ldots, \rho_{n+m, 0} \in[0,1]$, and $\pi^{1, s, d}, \ldots, \pi^{n+m, s, d} \in[0,1] \quad(s \in \mathcal{S}, d \in \mathcal{D})$, there exists a unique admissible weak solution, in the sense of Definition 2.4, $\rho=\left(\rho^{1}, \ldots, \rho^{n+m}\right)$ and traffic-type functions $\left(\pi^{1}(\cdot, \cdot, s, d), \ldots, \pi^{n+m}(\cdot, \cdot, s, d)\right)$ at the junction $J$ such that

$$
\begin{gathered}
\rho^{1}(0, \cdot) \equiv \rho_{1,0}, \ldots, \rho^{n+m}(0, \cdot) \equiv \rho_{n+m, 0}, \\
\pi^{1}(0, \cdot, s, d)=\pi^{1, s, d}, \ldots, \pi^{n+m}(0, \cdot, s, d)=\pi^{n+m, s, d}, \quad(s \in \mathcal{S}, d \in \mathcal{D}) .
\end{gathered}
$$

Moreover for every $i \in\{1, \ldots, n\}$ there exists $\hat{\rho}_{i}$ such that the solution for the density in $I_{i}$ is given by the wave generated by the Riemann problem with initial data $\left(\rho_{i, 0}, \hat{\rho}_{i}\right)$, while for every $j \in\{n+1, \ldots, n+m\}$ there exists $\hat{\rho}_{j}$ such that the solution for the density in $I_{j}$ is given by the wave generated by the Riemann problem with initial $\operatorname{data}\left(\hat{\rho}_{j}, \rho_{j, 0}\right)$. 
Moreover $\pi^{i}(t, \cdot, s, d)=\pi^{i, s, d}$ for every $t \geq 0, i \in\{1, \ldots, n\}, s \in \mathcal{S}, d \in \mathcal{D}$ and

$$
\pi^{j}\left(t, a_{j}+, s, d\right)=\frac{\sum_{i=1}^{n} \pi^{i, s, d} f\left(\hat{\rho}_{i}\right)}{f\left(\hat{\rho}_{j}\right)}
$$

for every $t \geq 0, j \in\{n+1, \ldots, n+m\}, s \in \mathcal{S}, d \in \mathcal{D}$.

Proof. In $\mathbb{R}^{n}$, define $r$ to be the linear subspace

$$
\left\{\begin{array}{l}
\gamma_{n}=p_{1} \gamma_{1}, \\
\vdots \\
\gamma_{n}=p_{n-1} \gamma_{n-1},
\end{array}\right.
$$

which is clearly a line in $\mathbb{R}^{n}$ by Definition 2.5. Consider the function $E: \mathbb{R}^{n} \rightarrow \mathbb{R}$ defined by

$$
E\left(\gamma_{1}, \ldots, \gamma_{n}\right)=c_{2} \sum_{i=1}^{n} \gamma_{i}-c_{1}\left[\operatorname{dist}\left(\left(\gamma_{1}, \ldots, \gamma_{n}\right), r\right)\right]^{2},
$$

where $\operatorname{dist}(\cdot, r)$ denotes the usual euclidean distance in $\mathbb{R}^{n}$ from $r$. Moreover, as in [8], we define the sets

$$
\begin{gathered}
\Omega_{i}:=\left\{\begin{array}{ll}
{\left[0, f\left(\rho_{i, 0}\right)\right],} & \text { if } 0 \leq \rho_{i, 0} \leq \sigma, \\
{[0, f(\sigma)],} & \text { if } \sigma \leq \rho_{i, 0} \leq 1,
\end{array} \quad i=1, \ldots, n,\right. \\
\Omega_{j}:=\left\{\begin{array}{ll}
{[0, f(\sigma)],} & \text { if } 0 \leq \rho_{j, 0} \leq \sigma, \\
{\left[0, f\left(\rho_{j, 0}\right)\right],} & \text { if } \sigma \leq \rho_{j, 0} \leq 1,
\end{array} \quad j=n+1, \ldots, n+m,\right.
\end{gathered}
$$

and

$$
\Omega:=\left\{\left(\gamma_{1}, \ldots, \gamma_{n}\right) \in \Omega_{1} \times \cdots \times \Omega_{n}: A \cdot\left(\gamma_{1}, \ldots, \gamma_{n}\right)^{T} \in \Omega_{n+1} \times \cdots \times \Omega_{n+m}\right\},
$$

where the entries $\alpha_{j, i}$ of the matrix $A$ are given by (2.6). The set $\Omega$ is clearly convex, compact and not empty. To define the solution to the Riemann problem at $J$ we have to solve the maximization problem

$$
\sup _{\left(\gamma_{1}, \ldots, \gamma_{n}\right) \in \Omega} E\left(\gamma_{1}, \ldots, \gamma_{n}\right) .
$$

Since $E$ is a continuous function and $\Omega$ is a compact set, the maximization problem admits a solution. Let us suppose that $\left(\hat{\gamma}_{1}, \ldots, \hat{\gamma}_{n}\right) \in \Omega$ and $\left(\tilde{\gamma}_{1}, \ldots, \tilde{\gamma}_{n}\right) \in \Omega$ satisfy

$$
E\left(\hat{\gamma}_{1}, \ldots, \hat{\gamma}_{n}\right)=E\left(\tilde{\gamma}_{1}, \ldots, \tilde{\gamma}_{n}\right)=\sup _{\left(\gamma_{1}, \ldots, \gamma_{n}\right) \in \Omega} E\left(\gamma_{1}, \ldots, \gamma_{n}\right)
$$

The Hessian matrix of $E$ is clearly equal to the Hessian matrix of the function

$$
-c_{1}\left[\operatorname{dist}\left(\left(\gamma_{1}, \ldots, \gamma_{n}\right), r\right)\right]^{2},
$$

since the term $c_{2} \sum_{i=1}^{n} \gamma_{i}$ is linear. If $\left(\nu_{1}, \ldots, \nu_{n}\right)$ is an orthogonal system where the first coordinate has the same direction of $r$, then the Hessian matrix of (3.9) has the form

$$
-2 c_{1}\left(\begin{array}{cccc}
0 & 0 & \cdots & 0 \\
0 & & \\
\vdots & I_{n-1} & \\
0 & &
\end{array}\right),
$$


where $I_{n-1}$ is the $(n-1) \times(n-1)$ identity matrix. Clearly (3.10) is a semi-negative definite matrix. This analysis shows that if $z_{1}, z_{2} \in \mathbb{R}^{n}, z_{1} \neq z_{2}$ and the line through $z_{1}$ and $z_{2}$ is not parallel to $r$, then

$$
E\left(\lambda z_{1}+(1-\lambda) z_{2}\right)>\lambda E\left(z_{1}\right)+(1-\lambda) E\left(z_{2}\right)
$$

for every $\lambda \in(0,1)$.

Suppose by contradiction that $\left(\hat{\gamma}_{1}, \ldots, \hat{\gamma}_{n}\right) \neq\left(\tilde{\gamma}_{1}, \ldots, \tilde{\gamma}_{n}\right)$. If the line through these two points is parallel to $r$, then (3.5) and (3.8) give

$$
c_{2} \sum_{i=1}^{n}\left(\hat{\gamma}_{i}-\tilde{\gamma}_{i}\right)=0
$$

and so $\left(\hat{\gamma}_{1}, \ldots, \hat{\gamma}_{n}\right)=\left(\tilde{\gamma}_{1}, \ldots, \tilde{\gamma}_{n}\right)$ since $r$ intersects the hyperplane $\sum_{i=1}^{n} \gamma_{i}=0$ just in the origin. Therefore (3.8) implies that the line through $\left(\hat{\gamma}_{1}, \ldots, \hat{\gamma}_{n}\right)$ and $\left(\tilde{\gamma}_{1}, \ldots, \tilde{\gamma}_{n}\right)$ is not parallel to $r$ and so (3.11) gives

$$
\begin{aligned}
E\left(\lambda\left(\hat{\gamma}_{1}, \ldots, \hat{\gamma}_{n}\right)+(1-\lambda)\left(\tilde{\gamma}_{1}, \ldots, \tilde{\gamma}_{n}\right)\right) & >\lambda E\left(\hat{\gamma}_{1}, \ldots, \hat{\gamma}_{n}\right)+(1-\lambda) E\left(\tilde{\gamma}_{1}, \ldots, \tilde{\gamma}_{n}\right) \\
& =\sup _{\left(\gamma_{1}, \ldots, \gamma_{n}\right) \in \Omega} E\left(\gamma_{1}, \ldots, \gamma_{n}\right)
\end{aligned}
$$

for every $\lambda \in(0,1)$, which is a contradiction. Therefore $\left(\hat{\gamma}_{1}, \ldots, \hat{\gamma}_{n}\right)$ is equal to $\left(\tilde{\gamma}_{1}, \ldots, \tilde{\gamma}_{n}\right)$, i.e. the point of maximum of $E$ is unique.

For every $i \in\{1, \ldots, n\}$, we choose $\hat{\rho}_{i} \in[0,1]$ such that

$$
f\left(\hat{\rho}_{i}\right)=\hat{\gamma}_{i}, \quad \hat{\rho}_{i} \in \begin{cases}\left.\left.\left\{\rho_{i, 0}\right\} \cup\right] \tau\left(\rho_{i, 0}\right), 1\right], & \text { if } 0 \leq \rho_{i, 0} \leq \sigma, \\ {[\sigma, 1],} & \text { if } \sigma \leq \rho_{i, 0} \leq 1 .\end{cases}
$$

Assumptions on $f$ imply that $\hat{\rho}_{i}$ exists and is unique. Let

$$
\hat{\gamma}_{j} \doteq \sum_{i=1}^{n} \alpha_{j i} \hat{\gamma}_{i}, \quad j=n+1, \ldots, n+m,
$$

and $\hat{\rho}_{j} \in[0,1]$ be such that

$$
f\left(\hat{\rho}_{j}\right)=\hat{\gamma}_{j}, \quad \hat{\rho}_{j} \in \begin{cases}{[0, \sigma],} & \text { if } 0 \leq \rho_{j, 0} \leq \sigma, \\ \left\{\rho_{j, 0}\right\} \cup\left[0, \tau\left(\rho_{j, 0}\right)[,\right. & \text { if } \sigma \leq \rho_{j, 0} \leq 1 .\end{cases}
$$

Since $\left(\hat{\gamma}_{1}, \ldots, \hat{\gamma}_{n}\right) \in \Omega, \hat{\rho}_{j}$ exists and is unique for every $j \in\{n+1, \ldots, n+m\}$. Solving the Riemann Problem (see [6, Chapter 6]) on each road, the first claim is proved.

The speeds $v^{i}$ of the traffic-type functions are positive; hence

$$
\pi^{i}(t, \cdot, s, d)=\pi^{i}(0, \cdot, s, d)=\pi^{i, s, d}
$$

for every $t \geq 0, i \in\{1, \ldots, n\}, s \in \mathcal{S}, d \in \mathcal{D}$. Finally, if $t \geq 0, j \in\{n+1, \ldots, n+m\}, s \in \mathcal{S}$, $d \in \mathcal{D}$, then $\pi^{j}\left(t, a_{j}+, s, d\right)$ is the percentage of $\rho^{j}\left(t, a_{j}+\right)$ which corresponds to cars going from the source $s$ to the destination $d$. Therefore it corresponds to the ratio

$$
\frac{\sum_{i=1}^{n} \pi^{i, s, d} f\left(\hat{\rho}_{i}\right)}{f\left(\hat{\rho}_{j}\right)},
$$

that is the quantity of cars going from $s$ to $d$ over the global amount of cars in $I_{j}$. 
REMARK 3.1. Notice that it may happen that a single road is the unique constraint for the maximization problem (3.7). This is due to the fact that the level curves of the function $E$ are paraboloids and the solution to the maximization problem can be given by the tangent point to a level curve of $E$ with a face of the boundary of $\Omega$.

REMARK 3.2. The solution to the Riemann problem at junctions is different from those introduced in [7] and [8].

Indeed, the solution of the Riemann problem given in [8] requires an additional condition (C), necessary for uniqueness, and that junctions have not two incoming and one outgoing roads, while the solution in [7] is equal to that in [8] except for the fact that the case of a junction with two incoming and one outgoing road is covered.

We recall here for reader's convenience the statement of condition (C) of [8].

(C) Let $\left\{e_{1}, \ldots, e_{n}\right\}$ be the canonical basis of $\mathbb{R}^{n}$ and for every subset $V \subset \mathbb{R}^{n}$ indicate by $V^{\perp}$ its orthogonal. Define for every $i=1, \ldots, n, H_{i}=\left\{e_{i}\right\}^{\perp}$, i.e. the coordinate hyperplane orthogonal to $e_{i}$ and for every $j=n+1, \ldots, n+m$ let $\alpha_{j}=\left(\alpha_{j 1}, \ldots, \alpha_{j n}\right) \in \mathbb{R}^{n}$ and define $H_{j}=\left\{\alpha_{j}\right\}^{\perp}$. Let $\mathcal{K}$ be the set of indices $k=\left(k_{1}, \ldots, k_{\ell}\right), 1 \leq \ell \leq n-1$, such that $0 \leq k_{1}<k_{2}<\cdots<k_{\ell} \leq n+m$ and for every $k \in \mathcal{K}$ set $H_{k}=\bigcap_{h=1}^{\ell} H_{k_{h}}$. Letting $\mathbf{1}=(1, \ldots, 1) \in \mathbb{R}^{n}$, we assume that for every $k \in \mathcal{K}$,

$$
\mathbf{1} \notin H_{k}^{\perp} .
$$

The choice of [8] and [7] is not good for this paper for the following reasons.

1. Since the matrix $A$ is given by formula (2.6), to satisfy condition $(\mathrm{C})$ we would need to impose some assumptions on the traffic-type functions, which are very technical and not meaningful from the modelling point of view.

2. The solution to the Riemann problem at the junction does not depend in a continuous way from the coefficients $\pi$. Indeed a small variation on the coefficients of $A$ may create a big variation in the solution of a Riemann problem.

On the contrary, the solution given here does not require condition (C) on the matrix $A$ and satisfies the property that small changes in the coefficients of $A$ produce small changes in the solution for the fluxes.

REMARK 3.3. Clearly the solution to the Riemann problem at a junction involves also the values of traffic-type functions, since they determine the entries of the matrix $A$. More precisely, only the values of the traffic-type functions in incoming roads influence the matrix $A$, while the values of the traffic-type functions in outgoing roads are determined by the solution of the Riemann problem for the density.

REMARK 3.4. Condition 7 in Definition 2.5 implies that, for every Initial-Boundary Condition and $s \in \mathcal{S}, d \in \mathcal{D}$, at most one $i \in\{1, \ldots, n\}$ is such that $\pi^{i, s, d} \neq 0$. Therefore (3.3) can be rewritten in the following way: for every $s \in \mathcal{S}, d \in \mathcal{D}, j \in\{n+1, \ldots, n+$ $m\}$, there exists $i \in\{1, \ldots, n\}$ such that

$$
\pi^{j}\left(t, a_{j}+, s, d\right)=\frac{\pi^{i, s, d} f\left(\hat{\rho}_{i}\right)}{f\left(\hat{\rho}_{j}\right)} .
$$


3.1. Junctions with two incoming and two outgoing roads. Let us introduce the definition of equilibrium for a Riemann problem at a junction and the definition of genericity for an equilibrium.

DeFINITION 3.3. Let $J$ be a junction with $n$ incoming roads, say $I_{1}, \ldots, I_{n}$, and $m$ outgoing roads, say $I_{n+1}, \ldots, I_{n+m}$, and let $A$ be a fixed distributional matrix at $J$.

We say that $\left(\rho_{1}, \ldots, \rho_{n+m}\right)$ is an equilibrium at $J$ if the solution $\left(\hat{\rho}_{1}, \ldots, \hat{\rho}_{n+m}\right)$ to the Riemann problem at $J$ with the initial data $\left(\rho_{1}, \ldots, \rho_{n+m}\right)$ coincides with $\left(\rho_{1}, \ldots, \rho_{n+m}\right)$.

We say that $\left(\rho_{1}, \ldots, \rho_{n+m}\right)$ is a generic equilibrium to the Riemann problem at $J$ if the following two conditions are satisfied:

1. the set $\Omega$ defined in (3.6) is different from $\{(0, \ldots, 0)\}$;

2. either the solution to the maximization problem (3.7) belongs to the interior of one face of $\Omega$ or belongs to a vertex of $\Omega$ generated by the intersection of exactly $n$ faces of $\Omega$.

Fix a junction $J$ with two incoming roads $I_{1}, I_{2}$ and two outgoing ones $I_{3}$ and $I_{4}$, consider a distributional matrix

$$
A=\left(\begin{array}{cc}
\alpha & \beta \\
1-\alpha & 1-\beta
\end{array}\right)
$$

and suppose that $\alpha>\beta$ and $0<p_{1}<1$. We study in detail equilibria (i.e. constant solutions) for the Riemann problem at $J$ when a single road is the unique active constraint for the maximization problem (3.7). In this case the function $E: \mathbb{R}^{2} \rightarrow \mathbb{R}$, defined by

$$
E\left(\gamma_{1}, \gamma_{2}\right)=c_{2}\left(\gamma_{1}+\gamma_{2}\right)-c_{1}\left[\operatorname{dist}\left(\left(\gamma_{1}, \gamma_{2}\right), r\right)\right]^{2}
$$

can be explicitly rewritten in the form

$$
E\left(\gamma_{1}, \gamma_{2}\right)=-\frac{c_{1}}{1+p_{1}^{2}}\left(\gamma_{2}-p_{1} \gamma_{1}\right)^{2}+c_{2}\left(\gamma_{1}+\gamma_{2}\right)
$$

Dini's implicit function theorem implies that the maximum $\left(\bar{\gamma}_{1}, \bar{\gamma}_{2}\right)$ of $E$ over $\Omega$ satisfies:

1. $\bar{\gamma}_{2}=p_{1} \bar{\gamma}_{1}+\frac{\left(1+p_{1}^{2}\right) c_{2}}{2 c_{1}}$, provided the road $I_{1}$ is the only active constraint;

2. $\bar{\gamma}_{2}=p_{1} \bar{\gamma}_{1}-\frac{\left(1+p_{1}^{2}\right) c_{2}}{2 c_{1} p_{1}}$, provided the road $I_{2}$ is the only active constraint;

3. $\bar{\gamma}_{2}=p_{1} \bar{\gamma}_{1}+\frac{\left(1+p_{1}^{2}\right) c_{2}(\alpha-\beta)}{2 c_{1}\left(\alpha+p_{1} \beta\right)}$, provided the road $I_{3}$ is the only active constraint;

4. $\bar{\gamma}_{2}=p_{1} \bar{\gamma}_{1}+\frac{\left(1+p_{1}^{2}\right) c_{2}(\beta-\alpha)}{2 c_{1}\left(p_{1}+1-\alpha-p_{1} \beta\right)}$, provided the road $I_{4}$ is the only active constraint.

Moreover the axis for the parabolas which are level curves of $E$ in the $\left(\gamma_{1}, \gamma_{2}\right)$ coordinates is given by the line

$$
\gamma_{2}=p_{1} \gamma_{1}+\frac{c_{2}\left(1-p_{1}\right)}{2 c_{1}}
$$

Table 3.1 describes all the possible generic equilibria both for the Riemann solver introduced in this paper and for that in [8] (briefly C-G-P Riemann solver). Notice that equilibria with only one active constraint are not admissible for the Riemann solver in [8]. Moreover some other types of equilibria (3 and 7 in table 3.1) are not admissible for the Riemann solver in [8]. 


\begin{tabular}{|r|c|c|c|}
\hline & Active constraints & new Riemann solver & C-G-P Riemann solver \\
\hline 1 & $I_{1}$ & yes & no \\
\hline 2 & $I_{1}$ and $I_{2}$ & yes & yes \\
\hline 3 & $I_{1}$ and $I_{3}$ & yes & no \\
\hline 4 & $I_{1}$ and $I_{4}$ & yes & no \\
\hline 5 & $I_{2}$ & yes & yes \\
\hline 6 & $I_{2}$ and $I_{3}$ & yes & no \\
\hline 7 & $I_{2}$ and $I_{4}$ & yes & no \\
\hline 8 & $I_{3}$ & yes & yes \\
\hline 9 & $I_{3}$ and $I_{4}$ & yes & no \\
\hline 10 & $I_{4}$ & yes & \\
\hline
\end{tabular}

TABLE 3.1. Equilibria for the Riemann solver introduced in this paper and for the Riemann solver introduced in [8] when $\alpha>\beta$.

If we impose conditions on $f(\sigma)$, then not all cases can happen as shown by the next results.

LEMMA 3.4. If $f(\sigma)<\frac{c_{2}\left(1+p_{1}^{2}\right)(\alpha-\beta)}{2 c_{1} p_{1}\left(p_{1}+1-p_{1} \beta-\alpha\right)}$, the equilibria 5,7 and 10 in table 3.1 can not happen.

If $f(\sigma)<\frac{c_{2}\left(1+p_{1}^{2}\right)(\alpha-\beta)}{2 c_{1}\left(p_{1} \beta+\alpha\right)}$, the equilibria 1,3 and 8 in table 3.1 can not happen.

Proof. If $f(\sigma)<\frac{c_{2}\left(1+p_{1}^{2}\right)(\alpha-\beta)}{2 c_{1} p_{1}\left(p_{1}+1-p_{1} \beta-\alpha\right)}$, then the region between the lines

$$
\gamma_{2}=p_{1} \gamma_{1}-\frac{\left(1+p_{1}^{2}\right) c_{2}}{2 c_{1} p_{1}}
$$

and

$$
\gamma_{2}=p_{1} \gamma_{1}+\frac{\left(1+p_{1}^{2}\right) c_{2}(\beta-\alpha)}{2 c_{1}\left(p_{1}+1-\alpha-p_{1} \beta\right)}
$$

in the $\left(\gamma_{1}, \gamma_{2}\right)$ plane do not intersect $\Omega$ and so the first statement holds. In the same way the second statement is proved.

Corollary 3.5. If $f(\sigma)<\min \left\{\frac{c_{2}\left(1+p_{1}^{2}\right)(\alpha-\beta)}{2 c_{1} p_{1}\left(p_{1}+1-p_{1} \beta-\alpha\right)}, \frac{c_{2}\left(1+p_{1}^{2}\right)(\alpha-\beta)}{2 c_{1}\left(p_{1} \beta+\alpha\right)}\right\}$, then the Riemann solver introduced in this paper and that introduced in [8] have the same types of equilibria.

Finally, for a simple network consisting of a single junction, we get the following proposition.

Proposition 3.1. Let us consider a road network with just one junction $J$, two incoming roads $I_{1}$ and $I_{2}$ and two outgoing roads $I_{3}$ and $I_{4}$. Assume that in incoming roads there are not $\Pi$-waves, so that the matrix $A$ for the junction $J$ is fixed and given by (3.13). If $\alpha>\beta$ and $f(\sigma)<\min \left\{\frac{c_{2}\left(1+p_{1}^{2}\right)(\alpha-\beta)}{2 c_{1} p_{1}\left(p_{1}+1-p_{1} \beta-\alpha\right)}, \frac{c_{2}\left(1+p_{1}^{2}\right)(\alpha-\beta)}{2 c_{1}\left(p_{1} \beta+\alpha\right)}\right\}$, then all estimates in [8] for waves interacting with $J$ hold. Hence for every positive time $T>0$, an entropic solution on $[0, T]$ exists on the network.

Proof. By Corollary 3.5, we know that C-G-P Riemann solver and the Riemann solver introduced in this paper have the same kinds of equilibrium. A deeper analysis shows that an arbitrary initial datum $\left(\rho_{1,0}, \rho_{2,0}, \rho_{3,0}, \rho_{4,0}\right)$ for the density at $J$ 
produces the same solution $\left(\hat{\rho}_{1}, \hat{\rho}_{2}, \hat{\rho}_{3}, \hat{\rho}_{4}\right)$ both for the C-G-P Riemann solver and for the Riemann solver introduced in this paper. In fact the set $\Omega$, defined in (3.6) is clearly the same for the two Riemann solvers. Moreover each maximization procedure implies that the maximum is on the boundary of $\Omega$. The maximum, by hypotheses, can be only at a vertex of $\Omega$ and the vertex must be the same, since either the roads $I_{1}$ and $I_{2}$ or the roads $I_{1}$ and $I_{4}$ or the roads $I_{2}$ and $I_{3}$ or the roads $I_{3}$ and $I_{4}$ can be the active constraints, as shown in Table 3.1. The estimates in [8] depend only on the solution of the Riemann problem and not on the Riemann solver used. Therefore we conclude.

\section{Wave-front tracking algorithm}

In this section a wave-front tracking algorithm is given for admissible solutions $(\rho, \Pi)$ in the sense of Definition 2.7. As in [8], we use the approach of Bressan; see [6].

For every $s \in \mathcal{S}$ and $d \in \mathcal{D}$, along each road we need to solve the system

$$
\left\{\begin{array}{l}
\rho_{t}^{i}(t, x)+f^{i}\left(\rho^{i}(t, x)\right)_{x}=0, \\
\pi_{t}^{i}(t, x, s, d)+v^{i}\left(\rho^{i}(t, x)\right) \pi_{x}^{i}(t, x, s, d)=0 .
\end{array}\right.
$$

First one takes piecewise constant approximations, in $L^{1}, \rho_{n}^{i}, \pi_{n}^{i}$, of the initial data $\bar{\rho}^{i}, \bar{\pi}^{i}$.

Then we construct a solution for the density solving all the Riemann problems until an interaction between two $\rho$-waves or between a $\rho$-wave with a junction. Rarefactions are approximated by rarefaction shock fans always inserting the value $\sigma^{i}$ when possible. The speed of a rarefaction shock is set to be the value of $\left(f^{i}\right)^{\prime}$ at its left endpoint, with the exception that every rarefaction shock with endpoint $\sigma^{i}$ has zero speed. Then we construct the solution for the traffic-type functions on the same interval of times. If an interaction of a $\Pi$-wave with a junction occurs, then we come back to this interaction time, we consider the new distribution matrix at the junction and we recalculate the solution for the density until the first interaction time. Repeating this procedure inductively, we are able to construct a wave-front tracking approximate solution.

To achieve the construction one needs estimates on the number of waves and on the total variation of the solution.

The bound on the number of waves is immediate on roads and, due to finite speed of propagation, it follows easily also on the whole network. The estimate of the total variation is the more delicate issue and is based on some approximation procedures and on basic interaction estimates, shown in the next section.

\section{Basic estimates of interactions}

Let us consider an admissible network $(\mathcal{I}, \mathcal{F}, \mathcal{J}, \mathcal{S}, \mathcal{D}, \mathcal{R}, \mathcal{P})$. Without loss of generality, we assume that $\rho_{\max }^{i}=1$ and $f^{i}=f$ for every road of the network. Hence $\sigma^{i}=\sigma$ for every $i \in\{1, \ldots, n\}$. Moreover in this section we do the following assumption:

(A1) every junction $J \in \mathcal{J}$ has at most two incoming roads and at most two outgoing roads.

Let us consider an equilibrium $(\bar{\rho}, \bar{\Pi})$ on the whole network, that is an admissible solution constant in time.

DeFinition 5.1. Let $J$ be a junction and let us consider an equilibrium at $J$. We say that the equilibrium is of the first type if there are exactly two active constraints for the maximization problem (3.7) and the corresponding hyperplanes are not tangent to a level curve of $E$ at the equilibrium. 
We say that the equilibrium is of the second type if there is exactly one active constraint for the maximization problem (3.7).

We also assume for the rest of the section:

(A2) for every $J \in \mathcal{J}$, the equilibrium is generic.

REMARK 5.1. A generic equilibrium for the Riemann problem at a junction is either of the first type or of the second type. Other types of equilibria are not generic, because there is at least one active constraint redundant for the maximization problem (3.7).

For example, consider a junction $J$ with two incoming roads $I_{1}, I_{2}$ and two outgoing roads $I_{3}, I_{4}$, for which the matrix $A$ is given by

$$
\left(\begin{array}{ll}
\frac{1}{2} & \frac{1}{2} \\
\frac{1}{2} & \frac{1}{2}
\end{array}\right)
$$

and $0<p_{1}<1$. Let $\left(\rho_{1}, \rho_{2}, \rho_{3}, \rho_{4}\right)$ be an equilibrium such that

$$
\begin{aligned}
& 0<\rho_{1}<\sigma, \quad \sigma<\rho_{2}<1, \quad \sigma<\rho_{3}<1, \quad 0<\rho_{4}<\sigma, \\
& f\left(\rho_{1}\right)=\frac{1}{2}, \quad f\left(\rho_{2}\right)=\frac{p_{1}}{2}, \quad f\left(\rho_{3}\right)=f\left(\rho_{4}\right)=\frac{1+p_{1}}{2} .
\end{aligned}
$$

In this case the roads $I_{1}$ and $I_{3}$ are active constraints. Also $\left(\tilde{\rho}_{1}, \rho_{2}, \rho_{3}, \rho_{4}\right)$ with $\sigma<$ $\tilde{\rho}_{1}<1, f\left(\tilde{\rho}_{1}\right)=\frac{1}{2}$ is an equilibrium, but in the second case only the road $I_{3}$ is an active constraint. So the first equilibrium is not generic, while the second one is.

Our aim is to prove an existence result for a solution $(\rho, \Pi)$ in the case of a small perturbation of the equilibrium $(\bar{\rho}, \bar{\Pi})$.

We have to consider the following types of interactions:

T1. interaction of $\rho$-waves with $\rho$-waves on roads;

T2. interaction of $\rho$-waves with $\Pi$-waves on roads;

T3. interaction of $\Pi$-waves with $\Pi$-waves on roads;

T4. interaction of $\rho$-waves with junctions;

T5. interaction of $\Pi$-waves with junctions.

Interaction of type $\mathrm{T} 1$ is classical and the total variation of the density decreases. Interaction of type T3 can not happen since $\Pi$-waves travel with speed depending only on the value of $\rho$.

REMARK 5.2. Hypothesis (A2) is fundamental in the next analysis, since it permits a reduction in the number of events at junctions and moreover since it excludes the possibility that an outgoing road becomes saturated.

Hypothesis (A2) can be relaxed, but can not be totally eliminated. In fact, if an outgoing road becomes saturated, then some of the next estimates are false.

5.1. Interaction of type T2. Let us consider a road $I_{i}$. First we note that the characteristic speed of a density is smaller than the speed of a $\Pi$-wave, as the next lemma shows.

LEMmA 5.2. Let $\rho \in[0,1]$ be a density and let $\lambda(\rho)$ be its characteristic speed. Then $\lambda(\rho) \leq v(\rho)$ and the equality holds if and only if $\rho=0$.

Proof. By definition, the speed $v$ is strictly decreasing with respect to the density $\rho$ and the flux $f$ is given by $f(\rho)=\rho v(\rho)$. This implies that

$$
\lambda(\rho)=f^{\prime}(\rho)=v(\rho)+\rho v^{\prime}(\rho) \leq v(\rho) .
$$


Clearly, if $\rho=0$, then $\lambda(0)=v(0)$, while if $\rho>0$, then $\lambda(\rho)<v(\rho)$.

Lemma 5.3. Let us consider a shock wave connecting $\rho^{-}$and $\rho^{+}$. Then:

T1. $\lambda\left(\rho^{-}, \rho^{+}\right)<v\left(\rho^{-}\right)$;

T2. $\lambda\left(\rho^{-}, \rho^{+}\right) \leq v\left(\rho^{+}\right)$and the equality holds if and only if $\rho^{-}=0$.

Proof. We have $\rho^{-}<\rho^{+}$and so $v\left(\rho^{-}\right)>v\left(\rho^{+}\right)$. Thus the first inequality is a direct consequence of the second one. Moreover, the speed of a shock wave is given by the Rankine-Hugoniot condition

$$
\lambda\left(\rho^{-}, \rho^{+}\right)=\frac{f\left(\rho^{+}\right)-f\left(\rho^{-}\right)}{\rho^{+}-\rho^{-}} .
$$

Since $f(\rho)=\rho v(\rho)$, we have that

$$
\lambda\left(\rho^{-}, \rho^{+}\right) \leq v\left(\rho^{+}\right)
$$

if and only if

$$
\rho^{+} v\left(\rho^{+}\right)-\rho^{-} v\left(\rho^{-}\right) \leq \rho^{+} v\left(\rho^{+}\right)-\rho^{-} v\left(\rho^{+}\right),
$$

which is satisfied if and only if

$$
\rho^{-} v\left(\rho^{-}\right) \geq \rho^{-} v\left(\rho^{+}\right) .
$$

The last inequality is clearly true. Notice that if $\rho^{-} \neq 0$ all the previous inequalities are strict inequalities.

REMARK 5.3. If we have a shock wave $\left(\rho^{-}, \rho^{+}\right)$with $\rho^{-}>0$, then by the previous lemma a $\Pi$-wave can cross the $\rho$-wave from the left, since $v\left(\rho^{-}\right)>v\left(\rho^{+}\right)>\lambda\left(\rho^{-}, \rho^{+}\right)$, that is the speed of the $\Pi$-wave when $\rho=\rho^{-}$is strictly greater than the speed of the $\Pi$-wave when $\rho=\rho^{+}$, which is strictly greater than the speed of the $\rho$-wave given by the Rankine-Hugoniot condition; see figure 5.1.

If instead $\rho^{-}=0$, then a $\Pi$-wave can interact with the $\rho$-wave since $v(0)>$ $\lambda\left(\rho^{-}, \rho^{+}\right)$, but then the discontinuity in $\Pi$ travels with the same speed of the $\rho$-wave; see figure 5.2 .

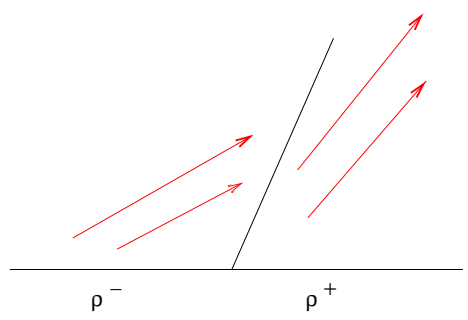

FIG. 5.1. Shock wave with $\rho^{-}>0$. The speed of the $\Pi$-waves is described by the arrows.

LEMma 5.4. Let us consider a rarefaction shock fan connecting $\rho^{-}$and $\rho^{+}$. Then $v\left(\rho^{+}\right)>v\left(\rho^{-}\right)>f^{\prime}\left(\rho^{-}\right)$.

Proof. We have $\rho^{-}>\rho^{+}$and so $v\left(\rho^{-}\right)<v\left(\rho^{+}\right)$. Moreover, $v\left(\rho^{-}\right)>f^{\prime}\left(\rho^{-}\right)$if and only if $v\left(\rho^{-}\right)>v\left(\rho^{-}\right)+\rho^{-} v^{\prime}\left(\rho^{-}\right)$and the last inequality is clearly true. 


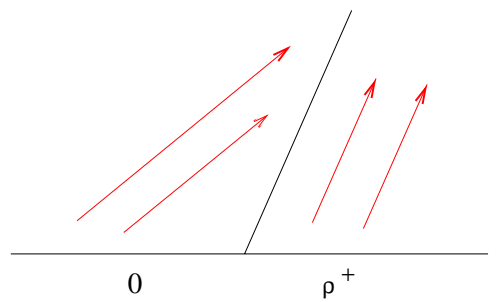

FIG. 5.2. Shock wave with $\rho^{-}=0$. The speed of the $\Pi$-waves is described by the arrows.

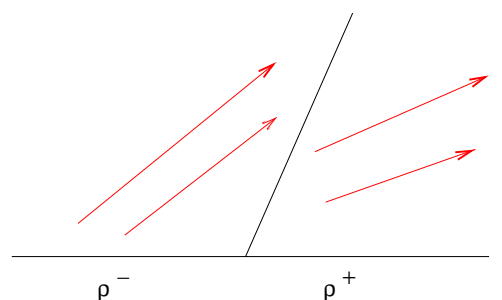

FIG. 5.3. Rarefaction shock fan. The speed of the $\Pi$-waves is described by the arrows.

REMARK 5.4. If we consider a rarefaction shock fan $\left(\rho^{-}, \rho^{+}\right)$, then the previous lemma shows that a $\Pi$-wave can cross the $\rho$-wave, since $v\left(\rho^{+}\right)$and $v\left(\rho^{-}\right)$are both strictly greater than the speed $f^{\prime}\left(\rho^{-}\right)$of the rarefaction shock fan; see figure 5.3.

REMARK 5.5. In principle, it is reasonable that the speed of a rarefaction fan can be chosen in the interval $\left[f^{\prime}\left(\rho^{-}\right), f^{\prime}\left(\rho^{+}\right)\right]$. Once we choose $f^{\prime}\left(\rho^{-}\right)$as the speed of a rarefaction fan, lemma 5.4 ensures that the speeds of $\Pi$-waves when $\rho=\rho^{-}$or $\rho=\rho^{+}$ are faster than the speed of the rarefaction fan.

If we choose another value $\lambda \in\left[f^{\prime}\left(\rho^{-}\right), f^{\prime}\left(\rho^{+}\right)\right]$for the speed of the rarefaction fan, then it may be happen that $v\left(\rho^{-}\right)<\lambda \leq v\left(\rho^{+}\right)$and this creates a problem to construct a wave front-tracking approximate solution for $\pi$; see figure 5.4.

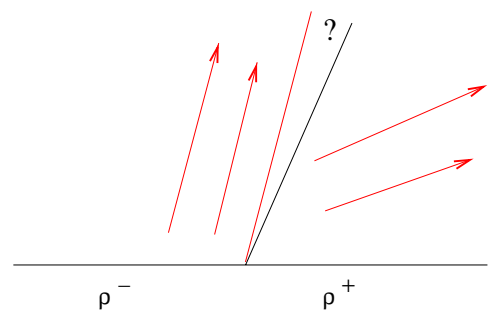

FIG. 5.4. Rarefaction shock fan with speed $\lambda>v\left(\rho^{-}\right)$. How to define a wave front-tracking approximate solution for the traffic distribution functions?

Putting together the previous lemmas we obtain the following result.

Proposition 5.1. An interaction of a $\rho$-wave with a $\Pi$-wave can happen only if the 
$\Pi$-wave interacts from the left respect to the $\rho$-wave. Moreover if this happens, then the $\rho$-wave does not change, while the $\Pi$-wave changes only its speed.

5.2. Interaction of type T4. We consider interactions of $\rho$-waves with junctions. In general these interactions produce an increment of the total variation of the flux and of the density in all the roads and a variation of the values of traffic-type functions on outgoing roads. As in [8], we can control the total variation of the flux. Indeed we have the following.

LEMMA 5.5. Let $J$ be a junction with at most two incoming roads and two outgoing ones. Let us suppose that a $\rho$-wave approaches the junction $J$ and assume (A2). If the total variation of the $\rho$-wave is sufficiently small, then there exists $C>0$, depending only on the values of the traffic-type functions on incoming roads, such that the total variation of the flux after the wave approaches $J$ is bounded by $C$ times the flux variation of the interacting wave, i.e.

$$
\text { Tot. Var. }{ }_{f}^{+} \leq \text {CTot. Var. } \cdot{ }_{f} .
$$

Proof. If the equilibrium at $J$ is of the first type, then the conclusion follows directly from the proof of Lemma 5.6 in [8].

Therefore assume that the equilibrium is of the second type. In this case only one road is an active constraint for the Riemann problem at $J$. If the total variation of the interacting wave is sufficiently small, then the wave modifies the equilibrium at $J$ if and only if it arrives from the road which is the active constraint, and the equilibrium type does not change, i.e. the constraint remains the same after the interaction. We consider only the case where the first incoming road $I_{1}$ is the active constraint, the other cases being similar. We denote by $\left(\gamma_{1,0}, \ldots, \gamma_{4,0}\right)$ the fluxes of the equilibrium at $J$, by $\gamma_{1}$ the flux of the interacting wave and by $\left(\hat{\gamma}_{1}, \ldots, \hat{\gamma}_{4}\right)$ the fluxes of the new equilibrium for the Riemann problem at $J$. As in subsection 3.1, we note that

$$
\gamma_{2,0}=p_{1} \gamma_{1,0}+\frac{\left(1+p_{1}^{2}\right) c_{2}}{2 c_{1}}, \quad \hat{\gamma}_{2}=p_{1} \hat{\gamma}_{1}+\frac{\left(1+p_{1}^{2}\right) c_{2}}{2 c_{1}}, \quad \gamma_{1}=\hat{\gamma}_{1}
$$

Thus

$$
\text { Tot.Var. }{ }_{f}^{-}=\left|\gamma_{1}-\gamma_{1,0}\right|
$$

and

$$
\text { Tot.Var. }{ }_{f}^{+}=\left|\hat{\gamma}_{2}-\gamma_{2,0}\right|+\left|\hat{\gamma}_{3}-\gamma_{3,0}\right|+\left|\hat{\gamma}_{4}-\gamma_{4,0}\right| \text {. }
$$

If $\gamma_{1}<\gamma_{1,0}$, then $\hat{\gamma}_{2}<\gamma_{2,0}, \hat{\gamma}_{3}<\gamma_{3,0}$ and $\hat{\gamma}_{4}<\gamma_{4,0}$. Therefore

$$
\begin{aligned}
\operatorname{Tot.Var}_{f}^{+}= & \left(\gamma_{2,0}-\hat{\gamma}_{2}\right)+\left(\gamma_{3,0}-\hat{\gamma}_{3}\right)+\left(\gamma_{4,0}-\hat{\gamma}_{4}\right) \\
= & \left(\gamma_{2,0}-\hat{\gamma}_{2}\right)+\alpha_{3,1}\left(\gamma_{1,0}-\gamma_{1}\right)+\alpha_{3,2}\left(\gamma_{2,0}-\hat{\gamma}_{2}\right) \\
& +\left(1-\alpha_{3,1}\right)\left(\gamma_{1,0}-\gamma_{1}\right)+\left(1-\alpha_{3,2}\right)\left(\gamma_{2,0}-\hat{\gamma}_{2}\right),
\end{aligned}
$$

since $\gamma_{3}=\alpha_{3,1} \gamma_{1}+\alpha_{3,2} \gamma_{2}$ and $\gamma_{4}=\left(1-\alpha_{3,1}\right) \gamma_{1}+\left(1-\alpha_{3,2}\right) \gamma_{2}$. Using (5.6) we conclude that

$$
\begin{aligned}
\text { Tot.Var. }{ }_{f}^{+} & =2\left(\gamma_{2,0}-\hat{\gamma}_{2}\right)+\left(\gamma_{1,0}-\gamma_{1}\right)=\left(1+2 p_{1}\right)\left(\gamma_{1,0}-\gamma_{1}\right) \\
& =\left(1+2 p_{1}\right) \text { Tot.Var. }{ }_{f}^{-} .
\end{aligned}
$$


If $\gamma_{1}>\gamma_{1,0}$, then the same calculation shows that

$$
\text { Tot.Var. }{ }_{f}^{+}=\left(1+2 p_{1}\right)\left(\gamma_{1}-\gamma_{1,0}\right)=\left(1+2 p_{1}\right) \text { Tot.Var. } ._{f}^{-} .
$$

This concludes the proof.

REMARK 5.6. Notice that the total variation of the flux can increase also when a wave interacts with a junction from an incoming road even if we start from an equilibrium of the first type. In fact, let us consider a junction $J$ with two incoming roads $\left(I_{1}\right.$ and $\left.I_{2}\right)$ and two outgoing ones $\left(I_{3}\right.$ and $\left.I_{4}\right)$ and we suppose that the active constraints are given by the roads $I_{1}$ and $I_{3}$. If a wave interacts with $J$ from $I_{1}$, changes the equilibrium configuration and the active constraints remain $I_{1}$ and $I_{3}$, then the total variation of the flux after the interaction is given by

$$
\text { Tot.Var. }{ }_{f}^{+}=\left|\hat{\gamma}_{2}-\gamma_{2,0}\right|+\left|\hat{\gamma}_{4}-\gamma_{4,0}\right|,
$$

where $\gamma_{i, 0}$ and $\hat{\gamma}_{i}(i \in\{1,2,3,4\})$ are the fluxes of the equilibrium respectively before and after the interaction. Since the active constraints before and after the interaction remain $I_{1}$ and $I_{3}$, we have that

$$
\alpha_{3,1} \gamma_{1,0}+\alpha_{3,2} \gamma_{2,0}=\alpha_{3,1} \hat{\gamma}_{1}+\alpha_{3,2} \hat{\gamma}_{2}
$$

and so

$$
\gamma_{2,0}-\hat{\gamma}_{2}=\frac{\alpha_{3,1}}{\alpha_{3,2}}\left(\hat{\gamma}_{1}-\gamma_{1,0}\right)
$$

Moreover

$$
\gamma_{4,0}=\left(1-\alpha_{3,1}\right) \gamma_{1,0}+\left(1-\alpha_{3,2}\right) \gamma_{2,0}, \quad \hat{\gamma}_{4}=\left(1-\alpha_{3,1}\right) \hat{\gamma}_{1}+\left(1-\alpha_{3,2}\right) \hat{\gamma}_{2},
$$

which implies

$$
\gamma_{4,0}-\hat{\gamma}_{4}=\left(1-\alpha_{3,1}\right)\left(\gamma_{1,0}-\hat{\gamma}_{1}\right)+\left(1-\alpha_{3,2}\right)\left(\gamma_{2,0}-\hat{\gamma}_{2}\right)
$$

Thus

$$
\text { Tot.Var. }{ }_{f}^{+}=\frac{\alpha_{3,1}+\left|\alpha_{3,2}-\alpha_{3,1}\right|}{\alpha_{3,2}} \text { Tot.Var. } \cdot{ }_{f}
$$

The conclusion follows from the fact that the coefficient

$$
\frac{\alpha_{3,1}+\left|\alpha_{3,2}-\alpha_{3,1}\right|}{\alpha_{3,2}}= \begin{cases}1, & \text { if } \alpha_{3,2} \geq \alpha_{3,1} \\ \frac{2 \alpha_{3,1}-\alpha_{3,2}}{\alpha_{3,2}}>1, & \text { if } \alpha_{3,2}<\alpha_{3,1}\end{cases}
$$

LEMma 5.6. Let $J$ be a junction with at most two incoming roads and two outgoing ones and assume (A2). Suppose that a $\rho$-wave approaches the junction $J$. If the total variation of the $\rho$-wave is sufficiently small, then there exists $C>0$ such that the variation of the traffic-type functions in outgoing roads is bounded by $C$ times the flux variation of the interacting wave, i.e.

$$
\text { Tot. Var } \cdot \stackrel{+}{\Pi} \leq \text { CTot. Var. } \cdot{ }_{f} .
$$


Proof. Fix a source $s \in \mathcal{S}$ and a destination $d \in \mathcal{D}$. We denote by $\pi_{i, 0}$ and $\hat{\pi}_{i}$ $(i \in\{1,2,3,4\})$ the values of the traffic-type functions for $s$ and $d$ at $J$, respectively, before and after the interaction of the $\rho$-wave with $J$.

If $\pi_{1,0}=\pi_{2,0}=0$, then clearly $\pi_{3,0}=\pi_{4,0}=0$ and $\hat{\pi}_{3}=\hat{\pi}_{4}=0$ and so we conclude.

Otherwise, since the path for each car is unique, we may assume $\pi_{1,0} \neq 0, \pi_{2,0}=0$, $\pi_{3,0} \neq 0$ and $\pi_{4,0}=0$. Since the speed of the traffic-type functions is positive, then $\hat{\pi}_{1}=\pi_{1,0}$ and $\hat{\pi}_{2}=0$. Moreover we have

$$
\pi_{3,0}=\pi_{1,0} \frac{\gamma_{1,0}}{\gamma_{3,0}}, \quad \hat{\pi}_{3}=\pi_{1,0} \frac{\hat{\gamma}_{1}}{\hat{\gamma}_{3}}, \quad \hat{\pi}_{4}=0
$$

where $\gamma_{i, 0}$ and $\hat{\gamma}_{i}$ denote the flux in road $I_{i}$ respectively before and after the interaction. Assumption (A2) implies that $\gamma_{3,0} \neq 0$. If the $\rho$-wave has sufficiently small total variation, then $\hat{\gamma}_{3} \neq 0$ and

$$
\left|\pi_{3,0}-\hat{\pi}_{3}\right|=\pi_{1,0}\left|\frac{\gamma_{1,0} \hat{\gamma}_{3}-\hat{\gamma}_{1} \gamma_{3,0}}{\hat{\gamma}_{3} \gamma_{3,0}}\right| \leq C \gamma_{1,0}\left|\hat{\gamma}_{3}-\gamma_{3,0}\right|+C \gamma_{3,0}\left|\hat{\gamma}_{1}-\gamma_{1,0}\right|
$$

for a suitable constant $C>0$. This permits to conclude using Lemma 5.5.

REMARK 5.7. The previous lemmas are proved under the assumption that the BV norm of the $\rho$-wave is such that the type of the equilibrium does not change. This excludes some realistic situations, for example that where an outgoing road becomes closed, due to the increment of the flow in the incoming roads.

5.3. Interaction of type T5. We consider interactions of $\Pi$-waves with junctions. Since $\Pi$-waves have always positive speed, they can interact with the junction only from an incoming road.

LEMMA 5.7. Let us consider a junction $J$ and a $\Pi$-wave on an incoming road $I_{i}$ interacting with $J$. If $A$ is the distributional matrix for $J$, whose entries are given by (2.6), then the interaction of the $\Pi$-wave with $J$ modifies only the $i$-th column of $A$. Moreover the variation of the $i$-th column is bounded by the $\Pi$-wave variation.

Proof. For each $s \in \mathcal{S}$ and $d \in \mathcal{D}$, we denote by $\pi_{i}^{s, d}$ and $\pi_{i, 0}^{s, d}$, respectively, the left and the right states of the $\Pi$-wave. Moreover, for every $j \in\{3,4\}$, we denote with $\alpha_{j, i}^{-}$ and $\alpha_{j, i}^{+}$, respectively, the entries of the matrix $A$ before and after the interaction of the $\Pi$-wave with $J$. By (2.6), it is clear that, if $l \neq i$, then the entries $\alpha_{j, l}$ are not modified. For $l=i$, we have

$$
\left|\alpha_{j, i}^{+}-\alpha_{j, i}^{-}\right| \leq \sum_{s \in \mathcal{S}, d \in \mathcal{D}, r_{J}(i, s, d)=j}\left|\pi_{i}^{s, d}-\pi_{i, 0}^{s, d}\right|
$$

This completes the proof.

REMARK 5.8. The previous lemma can be generalized to junctions with $n$ incoming roads and $m$ outgoing ones following the same proof.

LEMMA 5.8. Let us consider a junction $J$ and a $\Pi$-wave on an incoming road $I_{i}$ interacting with $J$. If the total variation of the $\Pi$-wave is sufficiently small, then there exists $C>0$ such that the variation of the fluxes is bounded by $C$ times the П-wave variation, i.e.

$$
\text { Tot. Var. }{ }_{f}^{+} \leq C \text { Tot. Var } \cdot \frac{-}{\Pi} \cdot
$$


Proof. We consider the case of two incoming and outgoing roads, the other cases being similar. Let us consider first the case of an equilibrium of the first type, i.e. there are exactly two roads that are active constraints for the Riemann problem at $J$. If the active constraints are given by the incoming roads $I_{1}$ and $I_{2}$, then the sets $\Omega_{1}, \Omega_{2}, \Omega_{3}, \Omega_{4}$ and $\Omega$, defined in the proof of Theorem 3.2, are given by

$$
\begin{gathered}
\Omega_{1}=\left[0, f\left(\rho_{1,0}\right)\right], \quad \Omega_{2}=\left[0, f\left(\rho_{2,0}\right)\right], \quad \Omega_{3}=\Omega_{4}=[0, f(\sigma)], \\
\Omega=\left\{\left(\gamma_{1}, \gamma_{2}\right) \in \Omega_{1} \times \Omega_{2}: A\left(\gamma_{1}, \gamma_{2}\right)^{T} \in \Omega_{3} \times \Omega_{4}\right\},
\end{gathered}
$$

where $\left(\rho_{1,0}, \rho_{2,0}, \rho_{3,0}, \rho_{4,0}\right)$ is the equilibrium for the density at $J$. Since the $\Pi$-wave is sufficiently small, then it does not affect the set $\Omega$. In fact, the perturbation of the $\Pi$-wave slightly modifies the constraints given by the lines

$$
\alpha_{3,1} \gamma_{1}+\alpha_{3,2} \gamma_{2}=f(\sigma) \quad \text { and } \quad \alpha_{4,1} \gamma_{1}+\alpha_{4,2} \gamma_{2}=f(\sigma) \text {. }
$$

These constraints remain non-active since

$$
\alpha_{3,1} f\left(\rho_{1,0}\right)+\alpha_{3,2} f\left(\rho_{2,0}\right)<f(\sigma) \quad \text { and } \quad \alpha_{4,1} f\left(\rho_{1,0}\right)+\alpha_{4,2} f\left(\rho_{2,0}\right)<f(\sigma) ;
$$

see figure 5.5. Hence the solution for the fluxes of the new Riemann problem at $J$ is given by

$$
\left(f\left(\rho_{1,0}\right), f\left(\rho_{2,0}\right), \alpha_{3,1}^{+} f\left(\rho_{1,0}\right)+\alpha_{3,2} f\left(\rho_{2,0}\right), \alpha_{4,1}^{+} f\left(\rho_{1,0}\right)+\alpha_{4,2} f\left(\rho_{2,0}\right)\right) .
$$

Therefore $\rho$-waves appear on $I_{3}$ and $I_{4}$ and the variation of the fluxes (and of the density) is proportional to the variation of the matrix $A$.

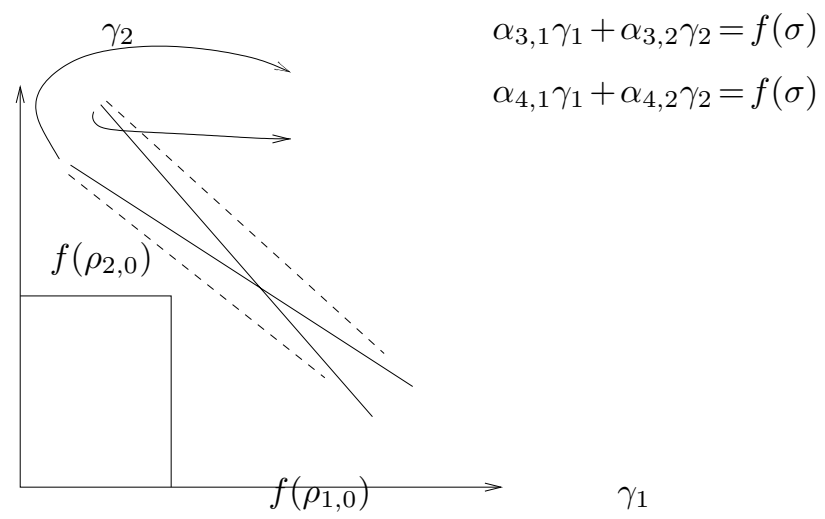

FIG. 5.5. The equilibrium when $I_{1}$ and $I_{2}$ are active constraints.

If the active constraints are one incoming road and one outgoing road or two outgoing roads, then the conclusion follows in an analogous way.

Let us consider now the case of an equilibrium of the second type. If an incoming road is the active constraint, then the equilibrium does not change for incoming roads 
$\alpha_{3,1} \gamma_{1}+\alpha_{3,2} \gamma_{2}=f\left(\rho_{3,0}\right)$

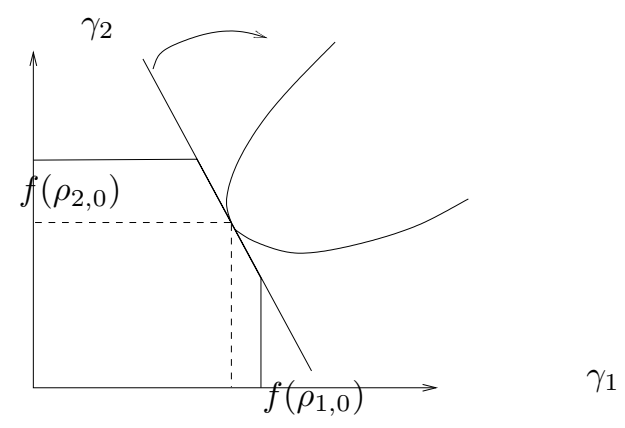

FIG. 5.6. The equilibrium when $I_{3}$ is the only active constraint. The axis are respectively $\gamma_{1}$ and $\gamma_{2}$. The point of maximum is $f\left(\rho_{1,0}\right)$ and $f\left(\rho_{2,0}\right)$ (in both the figures). Finally the arrows point to $\alpha_{3,1} \gamma_{1}+\alpha_{3,2} \gamma_{2}=f(\sigma), \alpha_{4,1} \gamma_{1}+\alpha_{4,2} \gamma_{2}=f(\sigma)$ in the first figure, while the arrow in figure 5.6 points to $\alpha_{3,1} \gamma_{1}+\alpha_{3,2} \gamma_{2}=f\left(\rho_{3,0}\right)$.

and the conclusion is as before. So we suppose that an outgoing road, say $I_{3}$, is the active constraint. It means that

$$
\Omega_{1}=\Omega_{2}=\Omega_{4}=[0, f(\sigma)], \quad \Omega_{3}=\left[0, f\left(\rho_{3,0}\right)\right] ;
$$

see figure 5.6. The change in the matrix $A$ affects the lines in $\mathbb{R}^{2}$

$$
\alpha_{3,1} \gamma_{1}+\alpha_{3,2} \gamma_{2}=f\left(\rho_{3,0}\right), \quad \alpha_{4,1} \gamma_{1}+\alpha_{4,2} \gamma_{2}=f(\sigma) .
$$

The new maximum point $\left(\hat{\gamma}_{1}, \hat{\gamma}_{2}\right)$ for the function $E$, defined in $(3.7)$, belongs to the line

$$
\alpha_{3,1}^{+} \gamma_{1}+\alpha_{3,2} \gamma_{2}=f\left(\rho_{3,0}\right),
$$

if the perturbation is sufficiently small. In fact, from the shape of the level curves of $E$, we deduce that $\left(\hat{\gamma}_{1}, \hat{\gamma}_{2}\right)$ is the tangent point of the previous line to a level curve of $E$. In our case the function $E$ is given by

$$
E\left(\gamma_{1}, \gamma_{2}\right)=\frac{1}{1+p_{1}^{2}}\left(\gamma_{2}-p_{1} \gamma_{1}\right)^{2}-\gamma_{1}-\gamma_{2}
$$

If we denote by $m$ the angular coefficient of

$$
\alpha_{3,1}^{+} \gamma_{1}+\alpha_{3,2} \gamma_{2}=f\left(\rho_{3,0}\right),
$$

i.e. $m=-\frac{\alpha_{3,1}^{+}}{\alpha_{3,2}}$, then the solution $\left(\hat{\gamma}_{1}, \hat{\gamma}_{2}\right)$ of the new Riemann problem for the fluxes in the incoming roads is given by the solution of the following system:

$$
\left\{\begin{array}{l}
\gamma_{2}-p_{1} \gamma_{1}=\frac{1+p_{1}^{2}}{2} \frac{1+m}{m-p_{1}}, \\
\alpha_{3,1}^{+} \gamma_{1}+\alpha_{3,2} \gamma_{2}=f\left(\rho_{3,0}\right),
\end{array}\right.
$$

where the first equation is the locus of the maximum point for $E$ when the only active constraint is given by $I_{3}$, while the second equation gives the expression of the active constraint. This permits us to conclude. 


\section{Perturbation of a solution}

Let us consider an admissible network $(\mathcal{I}, \mathcal{F}, \mathcal{J}, \mathcal{S}, \mathcal{D}, \mathcal{R}, \mathcal{P})$ and assume (A1), (A2) given in the previous section. We have the following theorem.

THEOREM 6.1. Let $(\bar{\rho}, \bar{\Pi})$ be an equilibrium on the whole network. For every $T>0$ there exists $\varepsilon>0$ such that the following holds. For every perturbation $(\tilde{\rho}, \tilde{\Pi})$ of the equilibrium with

$$
\|\tilde{\rho}\|_{B V} \leq \varepsilon, \quad\|\tilde{\Pi}\|_{B V} \leq \varepsilon
$$

and

$$
\|\tilde{\rho}-\bar{\rho}\|_{\infty}+\|\tilde{\Pi}-\bar{\Pi}\|_{\infty} \leq \varepsilon
$$

there exists an admissible solution $(\rho, \Pi)$ defined for every $t \in[0, T]$ such that at time $t=0$ coincides with $(\tilde{\rho}, \tilde{\Pi})$.

Proof. Let $\delta=\min _{I_{i}}\left(b_{i}-a_{i}\right), \hat{\lambda}=\max \left\{f^{\prime}(0),-f^{\prime}(1)\right\}$ and $N=\left[\frac{T \hat{\lambda}}{\delta}\right]+1$, where the brackets stands for the integer part. For every $\nu \in \mathbb{N}$, let $\hat{\rho}_{\nu}, \hat{\Pi}_{\nu}$ be two piecewise constant sequences approximating the initial conditions $\tilde{\rho}(0, \cdot), \tilde{\Pi}(0, \cdot)$ in BV-norm. We denote with $\rho_{\nu}^{*}, \Pi_{\nu}^{*}$ an approximate wave-front tracking solution on $[0, T]$ such that $\rho_{\nu}^{*}(0, \cdot)=\hat{\rho}_{\nu}(\cdot)$ and $\Pi_{\nu}^{*}(0, \cdot)=\hat{\Pi}_{\nu}(\cdot)$. For every interaction of a wave with a junction we have the estimates of Lemmas 5.5, 5.6, 5.8, if the strength of the waves are bounded by some $\bar{\varepsilon}>0$. Taking $\varepsilon=\frac{\bar{\varepsilon}}{N C}$ we get:

$$
\operatorname{Tot.} \operatorname{Var} . \Pi_{\nu}^{*}(t, \cdot) \leq N C \varepsilon=\bar{\varepsilon},
$$

and

$$
\text { Tot.Var. } f\left(\rho_{\nu}^{*}(t, \cdot)\right) \leq N C \varepsilon=\bar{\varepsilon}
$$

for every $t \in[0, T]$.

Theorem 5.10 in [8] implies that there exists $\rho^{*}$ such that $\rho_{\nu}^{*} \rightarrow \rho^{*}$ strongly in $L_{l o c}^{1}$, at least by extracting a subsequence. Moreover, by Helly theorem, there exists $\Pi^{*}$ such that $\Pi_{\nu}^{*} \rightarrow \Pi^{*}$ in $L_{l o c}^{1}$, at least by extracting a subsequence. We complete the proof with standard arguments.

\section{REFERENCES}

[1] D. Amadori and R. M. Colombo, Continuous dependence for 2x2 conservation laws with boundary, J. Differential Equations, 138, 229-266, 1997.

[2] F. Ancona and A. Marson, Scalar non-linear conservation laws with integrable boundary data, Nonlinear Analysis, 35, 687-710, 1999.

[3] A. Aw and M. Rascle, Resurrection of "Second Order" models of traffic flow?, SIAM J. App. Math., 60, 916-938, 2000.

[4] C. Bardos, A. Y. Le Roux and J. C. Nedelec, First order quasilinear equations with boundary conditions, Commun. Partial Differential Equations, 4, 1017-1034, 1979.

[5] S. Benzoni-Gavage and R. M. Colombo, An n-populations model for traffic flow, European J. Appl. Math., 14, 5, 587-612, 2003.

[6] A. Bressan, Hyperbolic Systems of Conservation Laws - The One-dimensional Cauchy Problem, Oxford Univ. Press, 2000.

[7] Y. Chitour and B. Piccoli, Traffic circles and timing of traffic lights for cars flow, Discrete Contin. Dynam. Systems, Series B 5, 3, 599-630, 2005. 
[8] G. M. Coclite, M. Garavello and B. Piccoli, Traffic flow on a road network, SIAM J. Math. Anal. 36, 6, 1862-1886, 2005.

[9] R. M. Colombo, Hyperbolic phase transitions in traffic flow, SIAM J. Appl. Math. 63, 2, 708$721,2002$.

[10] L. C. Edie, D. C. Gazis, W. Helly, R. Herman and R. Rothery, Third international symposium on the theory of traffic flow, Oper. Res. 13, 6, 1045-1051, 1965.

[11] M. Garavello and B. Piccoli, Traffic flow on a roads network using the Aw-Rascle model, accepted by Comm. Partial Differential Equations.

[12] J. M. Greenberg, Extension and amplifications of a traffic model of Aw and Rascle, SIAM Jour. Appl. Math. 62, 729-745, 2001.

[13] J. M. Greenberg, A. Klar and M. Rascle, Congestion on multilane highways, SIAM Jour. Appl. Math. 63, 3, 818-833, 2003.

[14] H. Holden and N. H. Risebro, A mathematical model of traffic flow on a network of unidirectional roads, SIAM J. Math. Anal., 26, 999-1017, 1995.

[15] J. P. Lebacque, The Godunov scheme and what it means for first order traffic flow models, in Transportation and Traffic Theory, Proceeding of the 13th ISTTT (J.B. Lesort ed.), Pergamon, Oxford, 647-677, 1996.

[16] M. J. Lighthill and G. B. Whitham, On kinetic waves. II. theory of traffic flows on long crowded roads, Proc. Roy. Soc. London Ser. A, 229, 317-345, 1955.

[17] P. I. Richards, Shock waves on the highway, Oper. Res., 4, 42-51, 1956. 